\title{
Linx
}

Revue des linguistes de l'université Paris X Nanterre

$7 \mid 1995$

Saussure aujourd'hui

\section{Diachronie et linéarité}

\section{Michel Arrivé}

\section{OpenEdition}

Journals

Édition électronique

URL : http://journals.openedition.org/linx/1133

DOI : 10.4000/linx.1133

ISSN : 2118-9692

\section{Éditeur}

Presses universitaires de Paris Nanterre

\section{Édition imprimée}

Date de publication : 1 septembre 1995

Pagination : 139-145

ISSN : 0246-8743

\section{Référence électronique}

Michel Arrivé, «Diachronie et linéarité », Linx [En ligne], 7 | 1995, mis en ligne le 12 juillet 2012, consulté le 10 décembre 2020. URL : http://journals.openedition.org/linx/1133; DOI : https://doi.org/10.4000/ linx.1133

Ce document a été généré automatiquement le 10 décembre 2020.

Département de Sciences du langage, Université Paris Ouest 


\title{
Diachronie et linéarité
}

\author{
Michel Arrivé
}

1 Je commence par un aveu: je suis loin d'avoir tout lu de l'immense littérature métasaussurienne. À vrai dire, cet aveu, tout regrettable qu'il est, me coûte assez peu : qui, à la réserve peut-être de Rudolf Engler, peut se vanter d'avoir lu tout ce qui s'est écrit sur Saussure? Mon ignorance aura peut-être pour effet de me faire enfoncer une porte déjà ouverte. Tant pis : je ne vise pas l'originalité. Je soupçonne, à vrai dire, que si la porte a déjà été ouverte, elle l'a été assez discrètement. Car, sur le sujet que je me propose d'aborder, il serait inexact de dire que rien - même à se limiter à ce que j'ai lu - n'a été dit. Mais ce qui a été dit l'a été discrètement, presque fugitivement, en tout cas sans l'insistance qui me paraît s'imposer pour un problème fondamental. Un détail révélateur : dans ses excellents Principes de diachonie (1990), Peter Wunderli ne fait que le frôler, à deux reprises (p. 20 et 90), sans utiliser le nom de la linéarité (ni l'expression caractère linéaire), qui ne figure pas dans l'index.

Qu'a-t-on dit sur le sujet? Buyssens, dès 1942, puis Godel, en 1969, ont repéré que "Saussure utilise de deux manières très différentes la notion de temps, selon qu'il envisage la perspective diachronique ou la perspective synchronique: dans le premier cas, le temps est l'agent, plus précisément la condition nécessaire du changement; dans le second, c'est simplement l'espace du discours " (Godel, 1969 : 207).

C'est en effet ce qui semble émaner de la séparation entre les deux notions de diachronie et de linéarité. Mais cette séparation est-elle absolue ? Ne faut-il pas poser le problème de l'éventuelle relation - quelle que soit la forme de cette relation - entre la linéarité du signifiant (Godel reprend à son compte la métaphore spatialisante de Saussure, en parlant de «l'espace du discours») et la diachronie («la condition du changement», dans les termes de Godel)? Dissimulée sous l'aspect strictement technique de sa formulation, l'importance du problème peut ne pas apparaître immédiatement. Il ne s'agit pourtant de rien d'autre que du problème du temps chez Saussure. On peut le schématiser par la question suivante : y a-t-il, dans le $C L G^{1}$, deux conceptions différentes du temps, celui de la diachronie et celui de la linéarité ? Ou bien est-il possible de saisir une relation entre ces deux temps, voire de ramener à l'unité la conception saussurienne du temps? 
4 Mais avant d'aborder l'examen de cette question, il convient de faire, après plusieurs autres $^{2}$, une remarque sur la façon dont Saussure, aujourd'hui encore, est lu. Dans l'opinion commune reçue par les linguistes - et pas seulement par eux ${ }^{3}$ - flotte l'idée que Saussure, père fondateur du "structuralisme", s'installe dans un point de vue statique qui neutralise le temps. Il sera inutile, ici, de citer un témoignage, parmi cent autres possibles : chacun, je pense, a plusieurs exemples en tête.

5 Il y aurait lieu de s'interroger sur l'origine de cette rumeur. Elle est en tout cas en contradiction flagrante avec la lettre de l'enseignement saussurien. Le CLG consacre au moins autant de place à la diachronie qu'à la synchronie. Ne chicanons pas sur des nombres de pages: Wunderli, 1990, p. 1-2, l'a fait avec précision, sans omettre de tenir compte du fait que la linguistique diachronique intervient aussi dans l'importante quatrième partie, consacrée à la "Linguistique géographique » et dans la cinquième partie, qui vise la «Linguistique rétrospective». Ce qui compte, ce sont les prises de position explicites. Citons l'une des plus significatives :

«Le fait que le Temps intervient pour altérer la langue, comme il intervient pour altérer <ou modifier> toute chose, ne semble pas d'abord un fait bien grave pour les conditions où est placée la science linguistique. Et je dois ajouter que je ne vois qu'une infime proportion de linguistes, ou peut-être aucune, qui soit disposée ellemême à croire que la question du Temps crée à la Linguistique des conditions particulières, des difficultés particulières, des questions particulières, voire une question centrale et pouvant aboutir à scinder la Linguistique en deux sciences ». (N 23.6, Engler 1989, p. 175).

6 Texte qu'il faut comparer à celui qui lui a été substitué dans l'édition standard (CLG, p. 114) : abrégé, affadi, il a entièrement perdu la " gravité » que Saussure, originellement, lui conférait, dans cet écrit - car il s'agit d'une note manuscrite en vue du troisième Cours. Saussure semble avoir fait sentir cette gravité dans l'énonciation orale de son enseignement : les notes de Constantin, pour ne parler que de lui, en conservent la trace. Une étrange pudeur semble avoir poussé les éditeurs à la censurer : ils ont escamoté, avec le rythme et les répétitions, tout ce qui est méditation sur le « $\mathrm{T}$ »emps (c'est intentionnellement que j'isole la majuscule entre guillemets: les éditeurs l'ont supprimée) - et non pas seulement sur le « facteur temps ».

7 Je reviens maintenant à la question posée. Pour l'aborder, on peut commencer par dire que la linéarité du signifiant est une propriété de la parole tandis que la diachronie affecte la langue. Les textes sont explicites, même si, pour la linéarité, il vaut mieux recourir aux sources manuscrites:
"Mais il y a ici un caractère capital de la matière phonique, non mis suffisamment en relief: c'est de se présenter à nous comme une chaîne acoustique, ce qui entraîne immédiatement le caractère temporel, qui est de n'avoir qu'une dimension. On pourrait dire que c'est un caractère linéaire : la chaîne de la parole, forcément, se présente à nous comme une ligne (souligné par MA), et cela a une immense portée pour tous les rapports postérieurs qui s'établiront. Les différences qualitatives (différence d'une voyelle à une autre, d'accent) n'arrivent à se traduire que successivement. On ne peut avoir à la fois une voyelle accentuée et atone; tout forme une ligne, comme d'ailleurs en musique» (Godel 1957, p. 205-206; Engler 1989$, p. 234 $)^{4}$.

8 Pour la diachronie, les textes prolifèrent : c'est bien la langue qui est affectée par elle, simultanément frappée d'immutabilité et de mutabilité, la première étant la condition de la seconde : 
«Le signe [élément de la langue, M.A.] est dans le cas de s'altérer parce qu'il se continue ». (CLG, p. 108-109)

En ce point, les choses paraissent simples. La linéarité est le mode d'intervention du temps dans la parole, la diachronie - ou, plus précisément, le changement diachronique - son mode d'intervention dans la langue. Mais ces deux modes d'intervention entrentils en relation? Au début, tout va bien : la notion de parole permet de jeter un pont entre linéarité et diachronie. Elle intervient en effet dans la définition même de la linéarité. Quant au changement diachronique, il trouve son origine dans la parole :

« Tout ce qui est diachronique dans la langue l'est par la parole, ne l'est que par la parole». (Godel 1957, p. 156 ; Engler 1989, p. 2235; voir aussi CLG, p. 138 - la formulation est exactement conforme à celle des sources manuscrites - et 143)

La diachronie serait donc la forme prise au niveau de la langue par ce qu'est la linéarité au niveau de la parole. Ainsi serait assurée la continuité entre les deux modes d'intervention du temps dans le langage : le temps subjectif du sujet énonçant, le temps objectif de la langue comme système. La linéarité serait la condition de la diachronie.

11 C'est sur le mot condition qu'il faut s'arrêter. Nécessaire, la condition de la linéarité ? Oui, à l'évidence : il faut bien qu'une langue soit parlée - c'est-à-dire donne lieu à des actes de parole, linéaires, temporalisés - pour qu'elle évolue. Mais suffisante? Eh bien non. Saussure envisage le problème sous la forme d'une spéculation, toute proche du mythe :

«Si l'on prenait la langue dans le temps, sans la masse parlante - supposons un individu isolé vivant pendant plusieurs siècles - on ne constaterait peut-être aucune altération; le temps n'agirait pas sur elle ». (CLG, p. 113)

12 La réflexion qui met en scène un individu parlant tout seul pendant plusieurs siècles paraît à première vue typiquement saussurienne. Engler 1989, p. 174, révèle qu'elle vient des éditeurs. Mais elle s'inscrit à mon sens parfaitement dans l'argumentation de Saussure. Quant au peut-être, qui atténue légèrement l'assertion, il figure bien dans les notes des auditeurs. Mais il est pratiquement effacé par la suite de l'argumentation, qui n'en tient plus le moindre compte. La formule capitale - «le temps n'agirait pas sur elle » - est pleinement catégorique ${ }^{6}$. Mais de quel temps s'agit-il ? Du temps « subjectif » de la linéarité, inséparable de tout acte de parole, qu'il y ait ou non « masse parlante $»^{7}$ ? Ou du temps «objectif» de la diachronie, qui entraîne les mutations linguistiques dès qu'intervient, en outre, la «masse parlante »? J'ai cru pouvoir, ailleurs ${ }^{8}$, considérer comme " évidente » l'interprétation de ce temps comme celui de la linéarité. Je ne vais pas jusqu'à me contredire, en choisissant le temps de la diachronie. Mais il m'apparaît maintenant que la décision est proprement impossible. C'est qu'en ce point se rencontrent, en un nœud définitivement fixé, les deux Temps saussuriens : celui de la linéarité de l'acte de parole - indispensable à l'évolution de la langue - et celui de la diachronie, qui n'est en somme que le même temps, dès qu'intervient la masse parlante.

On l'a compris: la duplicité de la conception saussurienne du temps n'est peut-être qu'apparente. Le seul facteur de séparation entre le temps de la linéarité et celui qui intervient dans l'évolution diachronique est la "masse parlante». Il suffit pour s'en convaincre de relire le passage du CLG p. 250 : la relation entre les deux proférations successives de Messieurs dans un même discours et celle qui s'établit entre pas (substantif) et pas (négation) ou entre calidum et chaud ne sont pas différentes : « Le second problème n'est en effet qu'un prolongement et une complication du premier ». Il y a en somme une seule identité des objets linguistiques à travers le temps, que ce temps soit celui de la 
linéarité ou celui de la diachronie. C'est donc que ces deux formes du temps n'ont pas à être distinguées.

Est-ce à dire que le problème est ainsi définitivement résolu ? Hélas non ! Et Wunderli qui, on l'a vu plus haut, n'envisage pas explicitement le problème des relations entre linéarité et diachronie - pose avec fermeté celui de l'identité diachronique. Il remarque justement les hésitations de Saussure, et va jusqu'à suggérer que la solution un moment adoptée - celle qui consiste à assimiler l'identité et la provenance - frôle " la tautologie » (1990, p. 54).

Mais il faut aller plus loin, et rappeler que la belle assurance affichée par le passage de la page 250 du CLG sur l'identité à lui-même du signe dans la linéarité du discours est loin d'être une constante de la pensée de Saussure. P.150, à propos des occurrences successives de Messieurs dans une conférence, il insiste sur les différences qui séparent ces réalisations, différences parfois "aussi appréciables que celles qui servent ailleurs à distinguer des mots différents" (p. 151). Il est plus explicite encore dans la Note 10 : "L'objet qui sert de signe n'est jamais 'le même' deux fois » (Engler 1990, p. 21).

Apparemment, la situation s'est complètement retournée. Si certains passages posent l'unicité du concept d'identité, d'autres en viennent à récuser toute possibilité, pour le signe, d'accéder à quelque identité que ce soit, synchronique ou diachronique. Contradiction? Certes, sur la conceptualisation de l'identité du signe. Et également sur l'effet du temps sur l'objet linguistique. Car dans la première conception, il laisse l'identité se maintenir, alors que dans la seconde il empêche de la poser'9

On remarquera toutefois que, paradoxalement, cette contradiction laisse entière la possibilité de maintenir l'unicité de la conception du temps saussurien. Car entre les deux positions contradictoires, il reste au moins quelque chose de commun : l'effacement de la différence entre temps de la linéarité et temps de la diachronie. Quel que soit son effet sur l'identité du signe, le temps intervient sans qu'il soit nécessaire (ou possible ?) d'en scinder la conception en temps de la linéarité du discours et temps de la diachronie.

C'est sur ce point que je crois pouvoir conclure. Provisoirement. Car il faudrait, pour formuler une opinion pleinement autorisée, alléguer les autres travaux de Saussure. Il s'agirait d'abord de la recherche sur la légende, où est posé le même problème. Faute de pouvoir entrer dans le détail, j'en dirai seulement que la notion d'identité dans ses relations avec le temps donne lieu à des analyses très voisines de celles qui, dans le CLG, mettent en cause jusqu'à la possibilité pour l'objet sémiotique (on sait qu'il prend dans la recherche le nom de symbole) d'accéder à l'identité ${ }^{10}$. Mais il conviendrait ensuite de revenir au Mémoire, et d' examiner la façon dont y est posé le problème de l'identité : les conclusions seraient sans doute assez différentes...

\section{BIBLIOGRAPHIE}

Arrivé, Michel, 1990, « Saussure : le temps et la symbolisation », in Sprachtheorie und Theorie der Sprachwissenschaft (Festschrift für Rudolf Engler), Tübingen, Gunter Narr, p. 37- 47. 
Buyssens, Éric, 1942, « Les six linguistiques de F. de Saussure », Les langues vivantes, $\mathrm{n}^{\circ}$ 1, p. 15-23 et $n^{\circ} 2$, p. 46-55.

Dosse, François, 1990, Histoire du structuralisme, tome I, Paris, La Découverte.

Engler, Rudolf, 1989, Ferdinand de Saussure. Cours de linguistique générale. Édition critique, tome I, Wiesbaden, Harrassowitz.

Engler, R., 1990, Ferdinand de Saussure. Cours [...], tome II, Wiesbaden, Harrassowitz.

Godel, Robert, 1969, Les sources manuscrites du Cours de linguistique générale de Ferdinand de Saussure, Genève, Droz.

Marinetti, Anna, et Meli, Marcello, 1986, Ferdinand de Saussure. Le leggende germaniche, Este, Zielo. Saussure, Ferdinand de, CLG, Cours de linguistique générale, Lausanne et Paris, Payot, 1916 (cité ici d'après l'édition de 1972).

Saussure, F. de, voir Engler 1989 et 1990.

Saussure, F de, voir Marinetti et Melo, 1986.

Wunderli, Peter, 1990, Principes de diachronie, Frankfurt am Main, Peter Lang.

\section{NOTES}

1. Et, ajouterai-je, dans l'ensemble de la réflexion proprement sémiologique de Saussure, c'est-àdire, selon moi, dans l'ensemble relativement homogène constitué par le CLG et la recherche sur la légende, à l'exclusion de la recherche sur les anagrammes, qui, toujours selon moi, échappe à la sémiologie dans le sens que lui donne Saussure. Voir dans ce volume mes deux autres interventions.

2. Notamment Wunderli 1990, p. 1 et passim.

3. Voir par exemple les 10 pages consacrées par Dosse 1991 (65-74), à la description de l'appareil théorique de Saussure: très significatives de ce que représente Saussure dans la $\delta o ́\} \alpha$ de l'intellectuel du XXème siècle finissant.

4. Je signale toutefois une minime difficulté textuelle que Godel n'a pas songé à signaler explicitement : ce passage des notes de Riedlinger - on se souvient qu'il n'a pas suivi le Cours III - est relatif à un fragment du Cours II, qui annonce les développements plus détaillés du Cours III sur « le caractère linéaire du signifiant». Les autres auditeurs du Cours II ont noté également que «la parole est bien représentée comme une ligne" (Gautier) ou que «la chaîne de la parole s'offre à nous comme une ligne » (Constantin) (Engler 1989, p. 234). En revanche, le syntagme la chaîne de la parole n'apparaît pas explicitement dans les notes des auditeurs du Cours III. Il reste que le caractère linéaire du signifiant est toujours, d'un Cours à l'autre, une propriété de la parole.

5. Godel a fait apparaître dans la même phrase la version donnée par Dégalier ( «l'est par la parole ») et de Mme Sechehaye («ne l'est que par la parole»). Les deux autres auditeurs ont entendu non l'est, mais « naît dans » (Joseph) ou « par la parole » (Constantin).

6. Celles des auditeurs le sont à des degrés divers. Mme Sechehaye va jusqu'à employer l'indicatif : «Le temps sans masse parlante ne produit rien » (Engler 1989, p. 174). Les autres, plus prudents, emploient le futur (Dégalier) ou le conditionnel (Constantin). La note 23.6, extrêmement elliptique, se contente de juxtaposer les deux formules opposées « Masse parlante sans Temps » et «Temps sans masse parlante».

7. Il faut en effet remarquer que Saussure n'exclut nullement l'idée d'un acte de parole individuel, sans «masse parlante». Voir notamment le passage de la Note 23.6 (Engler, 1989, 
p. 172) où est isolée « la partie [du langage] résidant dans l'âme de la masse parlante, ce qui n'est pas le cas pour la parole » (souligné par MA).

8. Arrivé, 1990, p. 42.

9. Est-il nécessaire de préciser que sur ce problème - qui, selon Saussure lui-même, excède les limites de la linguistique pour entrer dans le domaine de la «philosophie» - et sur les contradictions (apparentes ?) qu'il détermine dans la réflexion de Saussure, il est, ici, impossible de rien dire?

10. Voir Marinetti et Melo 1986, p. 30-31. 